\title{
Faster and Optimal Detection of Parametric Shapes
}

Shynimol E. Thayilchira

A project presented to Ryerson University in partial fulfillment of the requirement for the degree of Master of Engineering in the Program of Electrical and Computer Engineering.

Toronto, Ontario, Canada, 2007

(C) Shynimol Thayilchira, 2007 


\section{INFORMATION TO USERS}

The quality of this reproduction is dependent upon the quality of the copy submitted. Broken or indistinct print, colored or poor quality illustrations and photographs, print bleed-through, substandard margins, and improper alignment can adversely affect reproduction.

In the unlikely event that the author did not send a complete manuscript and there are missing pages, these will be noted. Also, if unauthorized copyright material had to be removed, a note will indicate the deletion.

\section{$\mathrm{UMI}^{\circ}$}

UMI Microform EC53579

\section{Copyright 2009 by ProQuest LLC}

All rights reserved. This microform edition is protected against unauthorized copying under Title 17, United States Code.

ProQuest LLC

789 East Eisenhower Parkway

P.O. Box 1346

Ann Arbor, MI 48106-1346 


\section{Author's Declaration}

I hereby declare that I am the sole author of this project.

I authorize Ryerson University to lend this project to other institutions or individuals for the purpose of scholarly research.

Shynimol Thayilchira 


\section{Abstract}

In this project, an analysis of the faster detection of shapes using Randomized Hough Transform (RHT) was investigated. Since reduced computational complexity and time efficiency are the major concerns for complex image analysis, the focus of the research was to investigate RHT for these specific tasks. Also, a detailed analysis of probability theory associated with RHT theory was investigated as well. Thus effectiveness of RHT was proven mathematically in this project. In this project, RHT technique combined with Generalized Hough Transform (GHT) using Newton's curve fitting technique was proposed for faster detection of shapes in the Hough Domain. Finally, the image under question was enhanced using Minimum Cross-Entropy Optimization to further enhance the image and then RGHT process was carried out. This helped the RGHT process to obtain the required time efficiency. 
This project is dedicated to my dearest daughters Fiona, Brionna and Riona and to my loving husband Babychen. 


\section{Acknowledgements}

I would like to thank my daughters and my husband Babychen for their constant support and understanding over the years. They are the ones who sacrificed their every day life while I was concentrating on my project work. Thanks a lot to my dear husband Babychen for his endless love and support through out my education. Many thanks to Dr. Krishnan for being so understanding and trying to help in all possible ways being a supervisor as well as a mentor. Special thanks to all my friends in Ryerson. 


\section{Contents}

1 Introduction 1

1.1 Motivation . . . . . . . . . . . . . . . 1

1.2 Objective and Specifications . . . . . . . . . . . 3

1.3 Technical and Functional specifications . . . . . . . . . 4

1.3.1 Technical System Requirements . . . . . . . . . . . . 4

1.3.2 Operating System . . . . . . . . . . . . . . . . 4

1.4 Technical Development Tools . . . . . . . . . . . 4

1.5 Report Organization ................. 5

2 Introduction to Hough Transform 6

2.1 Hough Transform . . . . . . . . . . . . . . 6

2.2 Theory of Hough Transform . . . . . . . . . . . 6

2.3 How does HT Work $\ldots \ldots \ldots \ldots \ldots \ldots$

2.4 Hough Transform Applications . . . . . . . . . . . . 8

2.5 Generalized Hough Transform (GHT) . . . . . . . . . . 9

2.6 Randomized Hough Transform (RHT) . . . . . . . . 10

2.7 Main difference between RHT and Conventional Hough Transform . . . . . . . . . . . . . . . 10

2.8 Mathematical Analysis of RHT . . . . . . . . . . . 11

2.9 Randomized Hough Transform for Straight Lines . . . . . . 12

2.10 Randomized Generalized Hough Transform (RGHT) using Newton's Curve Fitting technique . . . . . . . . . . . . 13

2.11 Wavelet Transform (WT) . . . . . . . . . . . . . 13

2.12 Theory of Discrete Wavelet Transform (DWT) . . . . . . . 14

2.12.1 Mathematical Explanation of DWT . . . . . . . 15

2.12.2 Decomposition Algorithm . . . . . . . . . . 15 
2.13 Applications of DWT . . . . . . . . . . . . . 17

2.13.1 Wavelet Decomposition of Images . . . . . . . . . . . 17

2.13.2 Advantages of Wavelet Decomposition ... . . . . . 17

2.14 Image Enhancement using minimum cross entropy Optimization 18 2.14.1 What is cross entropy minimization? . . . . . . 18

2.15 Iterative Solution to Obtain $\mathrm{Q}(\mathrm{t}, \mathrm{f}) \ldots \ldots \ldots \ldots$

3 Randomized Generalized Hough Transform (RGHT) for Straight Lines

3.1 Software Design . . . . . . . . . . . . . . . . . 21

3.2 Pseudo code for RGHT for Straight Lines . . . . . . . . 22

3.3 Analysis of Results . . . . . . . . . . . . . 23

4 Randomized Circular Hough Transform (RCHT) 25

4.1 RCHT Algorithm . . . . . . . . . . . . . 25

4.2 Pseudo code for RCHT . . . . . . . . . . . . 26

4.3 Simulation Results . . . . . . . . . . . . 27

5 RGHT For arbitrary shapes 28

5.1 RGHT For arbitrary shapes . . . . . . . . . . . 28

5.2 Pseudo code for RGHT for Arbitrary Shape . . . . . . . 30

5.3 Analysis of Results . . . . . . . . . . . . . 30

5.4 HWT for Arbitrary Shapes . . . . . . . . . . . . . 31

5.5 Image Enhancement $\ldots \ldots \ldots \ldots \ldots \ldots$

5.6 Observations . . . . . . . . . . . . . . . 33

5.7 Performance . . . . . . . . . . . . . . 33

6 Computational Complexity of Proposed Method 34

6.1 Computational Complexity of Proposed Method . . . . . . 34

6.2 Potential Applications of RGHT . . . . . . . . . . . 36

6.3 Conclusion ..................... 36

Bibliography 


\section{List of Figures}

1.1 Block diagram of the faster and optimal detection of parametric shapes .................. 3

2.1 Parametric description of a Straight Line . . . . . . . 8

2.2 Block diagram of the Wavelet Decomposition process . . . . 16

3.1 A Straight Line Image of 45 degree Inclination . . . . . . . . 23

$4.1185 \times 185$ pixels sample circular image $\ldots \ldots \ldots \ldots . \ldots 27$

5.1 An arbitray shape represented by the mathemaical equation $y=a o+a 1 *(x-x 1)+a 2 *(x-x 1) *(x-x 2)=1+1 *(x-$ $1)+1 *(x-1) *(x-1) \ldots \ldots \ldots \ldots$ 


\section{List of Tables}

5.1 RGHT time comparison table before and after decomposition . 32

5.2 RSLHT Results . . . . . . . . . . . . . . . . . 32

6.1 Comparison between RGHT and other Hough Transforms . . . 34

6.2 Time Complexity of HT related Algorithms . . . . . . . 35 


\section{Chapter 1}

\section{Introduction}

This chapter gives an introduction and overview of this project. The motivation, an overview of the project organization, objective and specifications, technical and functional specifications, technical development tools are the topics of this chapter.

\subsection{Motivation}

The motivation of the project is to perform a faster and optimal detection of parametric shapes using advanced signal and image analysis techniques. Hough Transform (HT) is a common technique for detecting shapes with parametric equation in an image. The classical Hough transform is most commonly used for the detection of regular curves such as lines, circles, ellipses, etc. A generalized Hough transform can be employed in applications where a simple analytical description of a feature(s) is not possible [1]. In order to apply the HT technique for complex shapes (GHT), a mathematical model or parametric forms for these shapes was developed using Newton's curve fitting technique [4]. Although complex shapes were successfully detected by this method, the computational complexity was substantially higher because of the large number of parameters (the number of parameters involved was 5 in this case) involved. In order to optimize the speed and computational 
time of GHT, Randomized Hough Transform (RHT) based on probabilistic approach was introduced [5].

RHT is based on the fact that a single parametric space can be determined uniquely with a pair, triple, or generally n-tuple of points from the original image [5]. Since random sampling is used, the algorithm works quite fast with low storage requirement. Due to the fact that one parameter point has to be solved for one n-tuple of points, computational complexity is less while comparing with GHT. However, current versions of RHT only work on binary images so that a preparation step of thresholding or filtering has to be applied to grayscale images beforehand. Moreover, it can only detect analytical geometric shapes and cannot be applied on arbitrary ones [5]. This leads to development of a method named Randomized Generalized Hough Transform(RGHT), which combines the RHT technique with already developed GHT technique.

RGHT combines the advantages of both GHT and RHT. It works on grayscale images and detects arbitrary grayscale object with various scales and orientations, like GHT. It adopts the random sampling mechanism and the converging mechanisms from $\mathrm{RHT}$ and thus reduces time and space complexities. It also detects arbitrary shapes as in the case of GHT. Moreover, it is based on probabilistic method as in RHT, so it has reduced time and space complexities, high accuracy and arbitrary resolution.

RGHT was able to detect any parametric shape with a high level of accuracy but in order to make it further faster, wavelet decomposition technique was applied. Arbitrary shape images particularly, high resolution images, occupy a lot of disc space. Discrete Wavelet transform (DWT) can be used to reduce the image size without losing much of the resolution. By applying the Wavelet decomposition technique, massive compression of the original image was obtained without significantly affecting resolution of the image. 
Considerable time efficiency was achieved by applying this method.

In order to make the above technique an optimal one, it was important to preprocess the image. Preprocessing the image is an important step in image processing. It is a proven result that image preprocessing produces more accurate results plus preprocessing is an important factor in achieving further time efficiency. In this project, image enhancement using minimum cross entropy optimization was performed to achieve better results plus in order to obtain time efficiency.

The first stage of HT is to convert the image to be analyzed to digital image format. In the second stage, HT is performed on the digitized image. Once the transformation is completed, in the third stage, peak detection process follows. Finally, the parameters of the shapes are computed. The following block diagram shows how the faster and optimal detection of parametric shapes were carried out in this project.

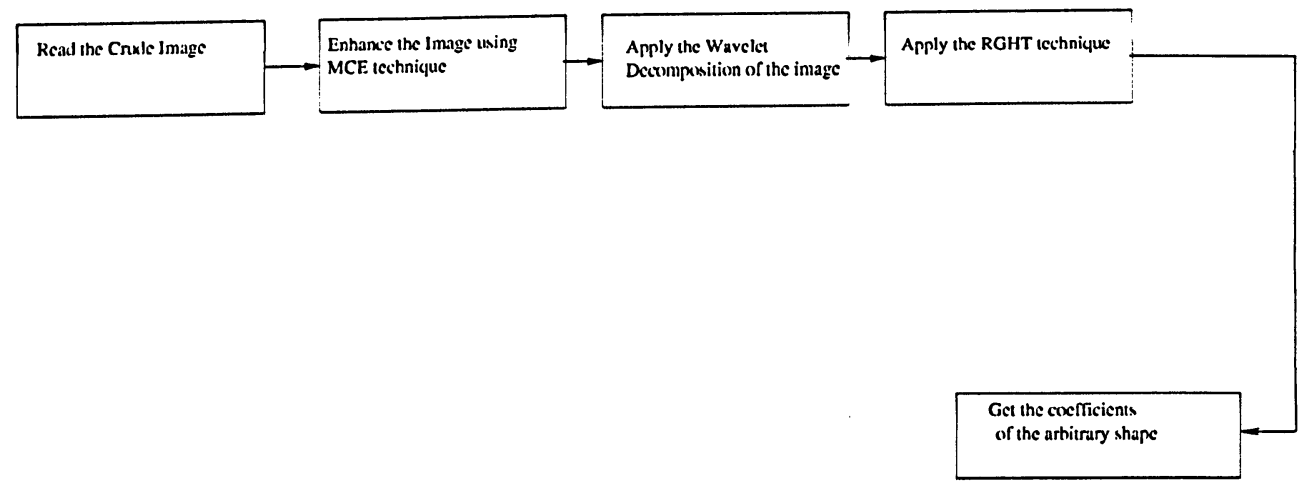

Figure 1.1: Block diagram of the faster and optimal detection of parametric shapes

\subsection{Objective and Specifications}

Objective: The main objective of this project is to investigate the real-time utility of RGHT in detecting complex shapes in an image as well as to make 
the detection faster and optimal. One way of achieving the time efficiency would be by combining HT and WT. By performing the image enhancement using the MCE technique before applying the RGHT technique would make the proposed method an optimal one.

Specifications: The specifications of the project are as follows:

1. Preprocessing the image to get accurate and faster results

2. Detection of simple shapes such as straight lines, circles etc. using HT.

3. Designing a parametric equation for arbitrary shape by using a curve fitting technique and then applying the HT.

4. Increasing the speed of detection by combining WT with the above technique.

\subsection{Technical and Functional specifications}

\subsubsection{Technical System Requirements}

Since time efficiency is the major concern in this project, it is desired to have faster computer systems. The operating system and other specifications of the project are given below:

\subsubsection{Operating System}

1. Linux: Fedora Core 5 with $700 \mathrm{MHz}$ frequency.

\subsection{Technical Development Tools}

Matlab Software Package version 7.2.0.29

1. Wavelet Toolbox 
2. Signal Processing Toolbox

3. Image Processing Toolbox

\subsection{Report Organization}

This project report is organized into six chapters. Chapter 1 is an introductory chapter about HT techniques, curve fitting technique as well as a brief demonstration on how the project is organized. Chapter 1 also describes the objectives and specifications for this project.Chapter 2 covers the terminology associated with $\mathrm{HT}$ as well as the theory behind HT. It also discuss applications of HT and how HT technique is used in the field of medicine. This chapter also explain the theory of Wavelet Transform as well as the theory of Discrete Wavelet Decomposition.Chapter 2 also describes the general theory of polynomial curve fitting, especially, Newton's general interpolation technique as well as the image enhancement technique using minimum cross entropy optimization.

Chapter 3 describes how Straight Line Hough Transform is implemented. This chapter also discusses the results of SLHT test. Chapter 4 briefly covers the theory and implementation of Circular Hough Transform (CHT).

Chapter 5 describes how RGHT for arbitrary shapes is implemented in this project.

Chapter 6 discusses the computational complexity of different HT algorithms and also the potential applications of RGHT. This chapter also includes the conclusion. 


\section{Chapter 2}

\section{Introduction to Hough Transform}

\subsection{Hough Transform}

Hough Transform (HT) is a simple and an elegant technique in detecting shapes. The first stage of $\mathrm{HT}$ is to convert the image to be analyzed to digital image form. In the second stage, HT is performed on the digitized image. Once the transformation is completed, in the third stage, peak detection process follows. Finally, the parameters of the shapes are found out.

\subsection{Theory of Hough Transform}

Before getting into the detailed theory of Hough Transform, some terminology associated with the Hough Transform has to be defined. Image plane is the X-Y plane where the original image exists. Parameter plane or Parameter space is where the transformed image is accumulated in an array of $n$ dimension. The dimension of the array depends on the type of the image to be analyzed. For example, for a straight line image, there are three parameters according to the method being adopted in this project, coefficients $a 0, a 1$ and $x 1$. The elements of the accumulator array correspond to small regions of parameter space. Prior to the actual transformation, there are some procedure to be considered in determining which pixel affects in representing the 
image. The process starts by setting a threshold, then comparing the pixel values with the threshold, and whichever passes the threshold measurement represents part of the image. At the same time, searching the location (row and column) of each pixel that represents the relative image. From these locations and the proper formulation of the image, the transformation process starts.

Basically, the transformation is from image space to parametric space. In the process of transforming, the parameter is stored in the accumulator. For example, if there are two parameters involved in the transformation, a two dimensional accumulator array is required. To start with, every element of the accumulator array is set to zero. For each point in parameter space to be recorded, the appropriate element of the array is incremented. Dividing parameter space up into regions is known as quantisation. Quantisation of the parameter space is usually done by trial and error method [18].

\subsection{How does HT Work?}

In order to illustrate how HT works, consider Straight Line Hough Transform (SLHT). Parameterization of SLHT involves transforming each point on the image space to $r$ and $\theta$ parameters as follows:

$$
x \cos (\theta)+y \sin (\theta)=r
$$

where $r$ is the length of the normal from the origin to this line and $\theta$ is the orientation of $\mathrm{r}$ with respect to the $\mathrm{X}$-axis. See figure 2.1. For any point $(\mathrm{x}, \mathrm{y})$ on this line, $\mathrm{r}$ and $\theta$ are constant. 


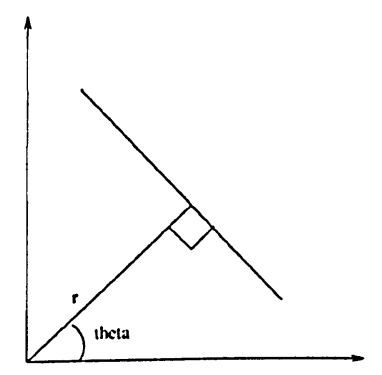

Figure 2.1: Parametric description of a Straight Line

From an image analysis context, the coordinates of the point(s) of edge segments i.e., $\left(x_{i}, y_{i}\right)$ in the image are known and, therefore, serve as constants in the parametric line equation, while $\mathrm{r}$ and $\theta$ are the unknown variables. If the possible $(r, \theta)$ values defined by each $\left(x_{i}, y_{i}\right)$ are plotted, points in cartesian image space map to curves (i.e. sinusoids) in the polar Hough parametric space. This point-to-curve transformation is the Hough transformation for straight lines. When viewed in Hough parameter space, points which are collinear in the cartesian image space become readily apparent as they yield curves which intersect at a common $(r, \theta)$ point. The transform is implemented by quantizing the Hough parameter space into finite intervals or accumulator cells(i.e. a multidimensional array). As the algorithm runs, each $\left(x_{i}, y_{i}\right)$ is transformed into a discretized curve and the accumulator cells which lie along this curve are incremented. Peaks in the accumulator array represent the corresponding straight line that exists in the image space.

\subsection{Hough Transform Applications}

Hough Transform (HT) has various practical applications such as:

1. Most manufactured parts (and many anatomical parts investigated in medical imagery) contain feature boundaries which can be described by 
regular curves or straight lines.

2. HT is commonly used in computer vision and pattern recognition for detecting geometric shapes that can be defined by parametric equations.

3. HT is commonly used in machine vision applications for detecting discontinuous patterns in noisy images [13].

4. HT finds many applications in astronomical data analysis.

5. HT is used to develop auto-adaptive, fast algorithms for the detection of echelle disorders and automated arc line identification [14].

\subsection{Generalized Hough Transform (GHT)}

Generalized Hough Transform (GHT)can be employed in applications where a simple analytic description of features is not possible. In order to apply the GHT technique, a mathematical model/equation has to be developed and HT technique is applied thereafter. In this project, the Newton's curve fitting technique has been applied to represent the arbitrary shape. Newton's general interpolating formula for data pairs $(x 1, y 1)$ through $(x n, y n)$ has the form, $y(x)=a_{0}+a_{1} *\left(x-x_{1}\right)+a_{2} *\left(x-x_{1}\right) *\left(x-x_{2}\right)+\ldots+$ $a_{n-1} *\left(x-x_{1}\right) *\left(x-x_{2}\right) \ldots .\left(x-x_{n-1}\right)$ where $a 0, a 1, \ldots$ are the coefficients. When considering the computational time involved in this process, only the coefficients $a 0, a 1, a 2$ were taken into consideration and the rest of the coefficients were ignored. In this case, the accumulator array is five dimensional which is $A(a 0, a 1, a 2, x 1, x 2)$. Even though complex shapes were successfully detected using this method, the computational complexity was very high because of the five dimensional accumulator array. In order to optimize the speed and computational time of GHT, Randomized Hough transform based on probabilistic approach was introduced. 


\subsection{Randomized Hough Transform (RHT)}

In RHT, a pair of pixels is mapped to a single cell in the parameter space. Because of this characteristic of RHT, it generates only a small subset of all parameter combinations. This characteristic of RHT is known as the many to one mapping or converging mapping.

\subsection{Main difference between RHT and Conventional Hough Transform}

The main difference between RHT and the conventional HT is that while in $\mathrm{HT}$ a single pixel in the original image is mapped to a curve in the parameter space, in RHT a pair of pixels is mapped to a single cell in the parameter space. Thus, while in HT curves are mapped into parameter space using a function which generates all parameter combinations compatible with both the observed pixel and the curve model, the RHT generates only a small subset of all parameter combinations. RHT Algorithm is described as below:

1. Form the set $P$ of all white points in an image.

2. Pick a point pair $(p i, p j)$ randomly from the set $P$.

3. Solve the parameter space point $(a, b)$ from the curve equation with points $(p i, p j)$.

4. Accumulate the cell $A(a, b)$ in accumulator space.

The first step can be done by scanning the original binary picture row-wise and storing the "white" pixels $p_{i}(x, y)$ to an array. Accumulation of the cell $A(a, b)$ means incrementing its value by one. A main task in the HT is to find the local maxima in the accumulator space, giving the parameters of the 
corresponding curves. In RHT, the local maxima can be easily detected one at a time. Maxima search can be done by the following method:

1. Run the RHT long enough to detect a global maximum in the accumulator space.

2. Remove all those points from the set $P$ which lie under the curve determined by the location of the maximum in the accumulator space.

3. Set the accumulator space to zero.

4. Continue to step 1.

The RHT algorithm stops when there are no more points left in the set $P$. The first step in the above algorithm contains a threshold which an accumulator point value must reach to be detected as the global maximum. The threshold must be selected depending on the original curve segment picture. Suitable values are 2 or 3 or even greater values if the original edge picture contains much noisy segments. Step 2 and 3 above, introduce an effective method to eliminate local maxima corresponding to noise or false curves. The points which lie under the detected curve can be found.

\subsection{Mathematical Analysis of RHT}

Mathematical modeling of RHT is based on the Generalized Bernoulli trials (GBT) of probability theory [7]. Bernoulli distribution is generally represented as

$$
p(x)=n C_{r} p^{x}(q)^{(n-x)},
$$

where $x$ is the number of successes in a total of $n$ trials and $p$ is the probability of success and $q=1-p$ is the probability of failure.

A sequence of identical and mutually independent trials each of which has 
$m+1$ possible outcomes: ' $m$ ' successes such as $T_{1}, T_{2}, ., T_{m}$ with corresponding probabilities $p_{1}, \ldots . p_{m}$ respectively and one type of failure $T_{f}$ with probability of failure $=1-($ probability of successes $)=1-\left(p_{1}+\ldots .+p_{m}\right)$.

Let $\xi_{1}, \xi_{2}, \ldots \ldots \ldots \xi_{m}$ denote the number of successes for $T_{1}, T_{2}, ., T_{m}$ respectively. In a sequence of $n$ GBT's, the multinomial (joint probability) distribution of $\xi_{1}, \xi_{2}, \ldots \ldots . \xi_{m}$ is

$$
P\left(\xi_{1}=k_{1}, \xi_{2}=k_{2}, \ldots \xi_{m}=k_{m}\right)=\frac{n !\left(p_{1}^{k_{1}} \ldots \ldots p_{m}^{k_{m}}\right) p_{f}^{k_{f}}}{k_{1} ! k_{2} ! \ldots . k_{m} ! k_{f} !}
$$

where $\sum_{1}^{m} k_{i}=n-k_{f}$.

In general, $\mathrm{P}\left(\xi_{i}=k_{i}\right)=n C_{k_{i}}\left(p_{i}^{k_{i}}\right) q_{i}^{n-k_{i}}$ where $q_{i}=n-p_{i}$ where $k_{i}=$ $0,1,2,3, \ldots n$ and $\xi_{i}$ satisfies the Binomial Distribution.

In this case, $E\left(\xi_{i}\right)=n p_{i}$ and $\sigma^{2}\left(\xi_{i}\right)=n p_{i} q_{i}$.

In an unlimited sequence of GBT's, let $\eta_{i}$ denote the total number of trials which precede (and include) the occurrence of the $r^{\text {th }}$ success of type $T_{i}$. Its Probability distribution satisfies the negative Binomial Distribution and can be written as,

$$
P\left(\eta_{i}=k_{i}\right)=\left(k_{i}-1\right) C_{(r-1)}\left(p_{i}\right)^{r} q^{k i-r}
$$

where $k_{i}=r, r+1 \ldots$.

In this case, expectation and variance becomes $E\left(\eta_{i}\right)=\frac{r}{p_{i}}$ and $\sigma^{2}\left(\eta_{i}\right)=$ $\frac{r\left(1-p_{i}\right)}{\left(p_{i}\right)^{2}}$

\subsection{Randomized Hough Transform for Straight Lines}

In order to illustrate how RHT works, let us take RHT for Straight Line into consideration. RHT for line detection uses the $y=m x+b$ concept, where $m$ is the slope of the line and $b$ is the $y$ intercept and $x$ and $y$ are the pixel coordinates. Since there are two parameters involved in the line detection, 2 points from the image has to be chosen randomly from the given straight 
line image and the RHT algorithm has to be applied for these two points. Let $p 1=(x 1, y 1)$ and $p 2=(x 2, y 2)$ be the randomly chosen points from the Straight Line image. Corresponding equation of the lines is

$$
\begin{aligned}
& y 1=a x 1+b \\
& y 2=a x 2+b .
\end{aligned}
$$

Solving these two equations determine the values of $\mathrm{a}$ and $\mathrm{b}$ and thus parameter space point $A(a, b)$ is obtained. This process is repeated until the maximum value in the accumulator reaches a certain threshold. The latest improvements in this area of research propose a new technique which combines RHT and GHT known as Randomized Generalized Hough Transform (RGHT) [6].

\subsection{Randomized Generalized Hough Transform (RGHT) using Newton's Curve Fitting technique}

In this project, RGHT using Newton's Curve Fitting technique is proposed for faster detection.It is well known that RHT works well for features with parametric equation. In case of features which lack a mathematical equation we have to fit a polynomial to get the coefficients. RGHT was implemented using the Newton's curve fitting technique ([16],[17]). Newton's general interpolating formula for data pairs $(x 1, y 1)$ through $(x n, y n)$ has the form $y(x)=a_{0}+a_{1} *\left(x-x_{1}\right)+a_{2} *\left(x-x_{1}\right) *\left(x-x_{2}\right)+\ldots .+a_{n-1} *\left(x-x_{1}\right) *\left(x-x_{2}\right) \ldots\left(x-x_{n-1}\right)$

\subsection{Wavelet Transform (WT)}

In order to optimize the time complexity of HT, Wavelet Transform was introduced. The Wavelet Transform (WT) provides a joint time-frequency 
representation. Passing the time-domain information through various high pass and low pass filters results in the output containing high and low frequency portions of the information. Next, by taking low pass portion of the filtered signal, the signal is put through the same filtering process as described above. This operation is called Decomposition. Decomposition process is continued until the signal reaches a pre-defined decomposition level [4].

\subsection{Theory of Discrete Wavelet Transform (DWT)}

Discrete wavelet transform (DWT), provides sufficient information both for analysis and synthesis of the original signal, with a significant reduction in the computation time. The DWT, decomposes the signal into a crude approximation and detail information. This method uses filters at different cutoff frequencies to analyze the signal at different scales. Furthermore, the signal is passed through a multiple selection of high pass filters to analyze the high frequencies. At the same time, the signal is passed through a series of low pass filters in order to analyze the low frequencies (decomposition process). DWT employs the following two sets of functions, Wavelet functions and Scaling functions, which are associated with high pass, and low pass filters respectively. The type of filtering that is applied to the signal, plays a major role in determining the resolution of the signal. Furthermore, the scale is changed by removing some of the samples of the applied signal (downsampling) or adding additional samples to the signal (upsampling). The resolution of the signal, which is a measure of the amount of information in the signal, is changed by the filtering operations, and the scale is changed by upsampling and downsampling (subsampling) operations.

Subsampling a signal corresponds to reducing the sampling rate, or removing some of the samples of the signal. Subsampling by a factor $\mathrm{n}$ reduces the number of samples in the signal $n$ times. Upsampling a signal corresponds to 
increasing the sampling rate of a signal by adding new samples to the signal. Upsampling a signal by a factor of $n$ increases the number of samples in the signal by a factor of $n$ ([4], [19]).

\subsubsection{Mathematical Explanation of DWT}

Assuming that the original signal $x[n]$ passes through a half band high pass filter $g[n]$, and through a low pass filter $h[n]$. Filtering a signal corresponds to the mathematical operation of convolution of the signal with the impulse response of the filter. Once filtering is completed, according to the Nyquist rule, half of the signal's samples are eliminated, resulting in signal's frequencies being $p i / 2$, rather than $p i$ as in the case of the original signal. Signal is subsampled by a factor of 2 , which results in a first level decomposition and is expressed by the equation:

$$
\begin{gathered}
Y_{\text {high }}[k]=\sum(x[n] * g[2 k-n]), \\
Y_{\text {low }}[k]=\sum(x[n] * h[2 k-n]),
\end{gathered}
$$

where $Y_{\text {high }}[k]$ and $Y_{\text {low }}[k]$ represent outputs of high pass and low pass filters. On one hand, the decomposition process halves the time resolution due to the fact that only half the number of samples now characterize the signal. On the other hand, this operation doubles the frequency resolution due to the fact that the frequency band of the signal after the decomposition, spans only half of the previous frequency band, effectively reducing the uncertainty in the frequency by half ([4], [19]).

\subsubsection{Decomposition Algorithm}

The above procedure, could be repeated for further decomposition. At every level, the filtering and subsampling resulted in half number of samples of the previous sample and half time resolution. At the same time, frequency band 
span halves and frequency resolution doubles. Decomposition algorithm is given below:

1. Pass the original signal through high pass and low pass filters and subsample by 2 .

Note: The output of the high pass filter after subsampling becomes the first level DWT coefficients.

2. Take the output of the low pass filter and then repeat step 1.

3. Repeat this procedure until two samples are left.

The following figure gives a detailed explanation of this algorithm. In this figure, $x[n]$ is the original signal to be decomposed, and $h[n]$ and $g[n]$ are lowpass and highpass filters, respectively. The bandwidth of the signal at every level is marked on the figure as $\mathrm{f}$.

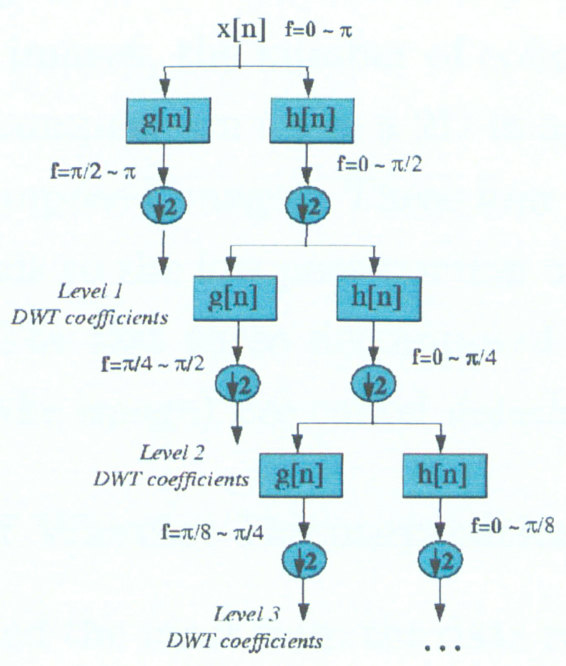

Figure 2.2: Block diagram of the Wavelet Decomposition process 


\subsection{Applications of DWT}

The concept of Wavelet decomposition can be applied to images or signals. Matlab commands 'dwt' and 'dwt2' performs the single level discrete 1D wavelet transform and single level discrete $2 \mathrm{D}$ wavelet transform of a signal respectively. Since images are of two dimensional nature, only 'dwt2' can be applied to an image in order to do a single level discrete 2D wavelet transform. Matlab commands 'wavedec' and 'wavedec2' performs multi level 1D wavelet transform and multi level $2 \mathrm{D}$ wavelet decomposition of a signal respectively [4].

\subsubsection{Wavelet Decomposition of Images}

In this project, the concept of Wavelet Decomposition is applied to images. Due to the fact that images are of two dimensional nature, only 'wavedec2' can be applied to an image in order to do a multi level 2D wavelet decomposition. In the decomposed images, the number of columns and rows reduce by a factor of 2. Wavelet decomposition takes a 2D image and convert it to four minimized standard decomposed images. These four decomposed images are: approximated (corresponds to the low pass portion of the image), horizontal, vertical, and diagonal. The last three decomposed images (corresponds to the high pass portion of the image) are called detailed information [4].

\subsubsection{Advantages of Wavelet Decomposition}

One area that has benefited the most from the data reduction property of the wavelet transforms is image processing. Images, particularly high-resolution images, occupy a lot of disk space. DWT can be used to reduce the image size without losing much of the resolution. When applying DWT on any image,it can be noted that massive compression of the original image is obtained 
without significantly affecting the resolution of the image.

\subsection{Image Enhancement using minimum cross entropy Optimization}

\subsubsection{What is cross entropy minimization?}

Cross-entropy minimization is a general method of inference about an unknown PDF when there exists a prior estimate of the function and new information in the form of constraints on expected values is available [10]. If the optimized / enhanced image constraint (an unknown PDF) is denoted by $Q(t, f)(Q(t, f)$ is a positive joint distribution), then it should satisfy the marginals

$$
\begin{gathered}
\int Q(t, f) d f=\left|S(t)^{2}\right|=m_{0}(t), \\
\int Q(t, f) d t=\left|S(f)^{2}\right|=m_{0}(f) .
\end{gathered}
$$

The above equations can be treated as constraint equations (new information) for optimization. Now $Q(t, f)$ may be obtained from $P^{\prime}(t, f)$ (a prior estimate of the function) by minimizing the cross entropy between them given by

$$
H\left(Q, P^{\prime}\right)=\iint Q(t, f) \log \left(\left(Q(t, f) / P^{\prime}(t, f)\right) d f d t\right.
$$

Lagrange multipliers can be used to solve for the $Q(t, f)$ that satisfies the above constraints and minimize the cross entropy between them. Thus

$$
Q(t, f)=P^{\prime}(t, f) \exp \left\{-\left(\lambda_{0}+\psi_{0}+\lambda_{1} f^{1}+\psi_{1} t^{1}+\lambda_{2} f^{2}+\psi_{2} t^{2}+\ldots+\lambda_{n} f^{n}+\psi_{n} t^{n}\right)\right\}
$$


where $\lambda_{i}$ and $\psi_{i}$ are Lagrange multipliers to be determined via the constraint equations.

\subsection{Iterative Solution to Obtain $Q(t, f)$}

At the "zeroth" iteration equation (2.13) becomes

$$
Q^{0}(t, f)=P^{\prime}(t, f) \exp \left(-\lambda_{0}\right) \text {. }
$$

$\lambda_{0}$ can be solved by imposing the time marginal constraint of (2.10) on (2.14).

$$
\lambda_{0}=\ln \left(\left(p^{\prime}(t) / m_{0}((t))\right.\right.
$$

where $m_{0}(t)$ is the true (desired) time marginal of $(2.10)$ and $\left.p^{\prime}(t)\right)$ is the time marginal of $P^{\prime}(t)$.

Substituting the value of $\lambda_{0}$ back into (2.14) gives

$$
\left.Q^{0}(t, f)=P^{\prime}(t, f)\left(m_{0}(t)\right) / p^{\prime}(t)\right)
$$

In this iteration $Q^{0}(t, f)$ has the correct time marginal but at this stage it need not contain the correct frequency margin. In order to obtain the correct frequency marginal $Q^{0}(t, f)$ is used as a new prior estimate in the next iteration.

$$
Q_{1}(t, f)=Q^{0}(t, f) \exp \left(-\psi_{0}\right)
$$

$\psi_{0}$ can be solved by imposing the time marginal constraint of (2.11) on (2.14)

$$
\psi_{0}=\ln \left(\left(q^{\prime}(f) / m_{0}((f))\right.\right.
$$

Substituting the value of $\psi_{0}$ back into (2.17) gives

$$
Q_{1}(t, f)=Q^{0}(t, f)\left(m_{0}(f) / q^{0}(f)\right)
$$


where $m_{0}(f)$ is the true(desired) frequency marginal constraint of (2.11) and $q^{0}(f)$ is the frequency marginal of $Q^{0}(t, f)$. After this iteration, $Q^{1}(t, f)$ yields the desired frequency marginal, but no longer contains the correct time marginal. By continuing the iteration helps to get a more accurate $Q(t, f)$,since $H\left(Q, Q^{(i+1)}\right)<H\left(Q, Q^{(i)}\right), Q^{(i+1)} \neq Q^{(i)}$ [because of the convexity of cross entropy]. This process is continued until convergence is reached. Convergence criterion is determined depending on the specific application[11]; at this point,the solution $Q^{N}(t, f)$ is the MCE-TFD $Q(\mathrm{t}, \mathrm{f})[12]$. 


\section{Chapter 3}

\section{Randomized Generalized Hough Transform (RGHT) for Straight Lines}

Straight Line Hough transform (SLHT) is one of the method utilized to transform points on straight line image to a corresponding parametric plane, which consists of two parameters. For an equation of a straight line, $y=$ $a 0+a 1(x-x 1), a 0$ and $a 1$ are the coefficients of the line. The SLHT transforms the X-Y form into $a 0$ and $a 1$ image parameter forms. These image parameter is accumulated in three - dimensional accumulator array $A(a 0, a 1, x 1)$. Furthermore, before computing the HT of straight line, the parameter space is quantized between appropriate maximum and minimum values of a0min and $a 0 \max$, and $a 1 \min$ and $a 1 \max$, and $x 1 \min$ and $x 1 \max$. Once quantization of the parameter space is done, the actual HT method can be implemented.

\subsection{Software Design}

In order to implement SLHT, the following steps are considered:

1. Scan the image and accumulate the image points in set $P$.

2. Initialize the accumulator array vote $(a 0, a 1, x 1)=0$. 
3. Pick two points randomly from the set $P$.

4. For each point $(x i, y i)$, vary a1 from 1 to the number of rows in the image, also vary $x 1$ from 1 to the number of columns in the image. Next, in the limited quantized plane, increment the number of counts each time a line passes through each cell until the transformation is completed. The number of counts in each cell of the parametric image are called votes, $A(a 0, a 1, x 1)=A(a 0, a 1, x 1)$.

5. Local maximum in the accumulator array corresponds to collinear points in the image plane. The accumulator array provides a measure of the number of points on the line. Algorithm is stopped when the accumulator array reaches a certain threshold value.

The next step is to search for the peak in the accumulator array. The peaks and their corresponding coordinates are used to identify potential lines within the image plane. These peaks provide the values of $a 0, a 1$ and $x 1$.

\subsection{Pseudo code for RGHT for Straight Lines}

1. First, digitize the original image.

2. Initialize the accumulator array to zero.

3. Save the values of the image pixel to a data set $P$.

4. Randomly pick two points from set $P$.

5. For each pixel that passes the certain threshold value, calculate the coefficient $a 0$ for all possible values of $a 1$ and $x 1$. The formula to calculate $a 0$ is given below: $a 0=y-a 1 *(x-x 1)$.

6. Then increment the accumulator array. 
7. end.

\subsection{Analysis of Results}

Using steps mentioned above, SLHT was applied on a 45 degree straight line (See figure 3.1).

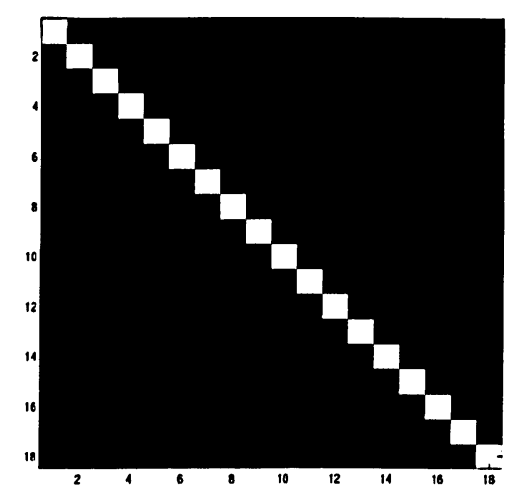

Figure 3.1: A Straight Line Image of 45 degree Inclination

For this straight line image, the coefficients are $a 0=1, a 1=1$ and $x 1=1$. From the SLHT program output, the maximum vote was found to be 3 points located at $a 0=18, a 1=1$ and $x 1=18$. Since the original equation of this straight line is

$$
y=a 0+a 1 *(x-x 1)=1+1 *(x-x 1)=x .
$$

It can be observed that the coefficient $a 1$ determines the characteristics of the line as well as the orientation of the line. For example if $a 1=1$, then the image in question is a straight line of the orientation of 45 degree. After carefully reviewing all possible values for $a 1$, it can be interpreted that the value of the coefficient $a 1$ determines the shape and orientation of the particular image. From the output of the SLHT, the equation of the line is found as

$$
y=18+1 *(x-18)=x
$$


which agrees with the original equation of the image as in Equation (3.2). Also note that $a 1$ values for the original image as well as the $a 1$ value obtained from the SLHT are same, thus confirming the result.

The intention is to extend the HT for other shapes as well and the next chapter describes the Randomized Circular Hough Transform. 


\section{Chapter 4}

\section{Randomized Circular Hough Transform (RCHT)}

As a follow up, RCHT has been implemented. For circles, the parameters were derived using the basic equation of the circle,

$$
(x-a)^{2}+(y-b)^{2}-r^{2}=0
$$

where $x$ and $y$ are image coordinates, $a$ and $b$ are location of the center, and $r$ is the radius. From the above equation, there are three parameters $a, b$, and $r$. For example, if the edge pixels of an image are arranged on a circle with parameters $a o, b o$, and $r o$, the resultant loci of the parameters for each such point passes through the same point $(a o, b o, r o)$. Thus, many such circles intersects at a common point. As in the case of SLHT, implementation of RCHT also involves a three dimensional array. From Equation (3.1), fix $x$ and $y$, and let $a, b$ and $r$ to vary.

\subsection{RCHT Algorithm}

The algorithm used to implement RCHT is given below:

1. Scan the image and accumulate the image points in set $P$.

2. Initialize the accumulator array vote $(a, b, r)=0$. 
3. Pick 3 points randomly from the set $P$.

4. For image exist in each pixel for fixed values of row value and $y$ value calculate radius $r$ of the circle for all possible values of $a$ and $b$. For each set of $(a, b, r)$ thus found, a vote is added to the corresponding cell of accumulator array. $A(a, b, r)=A(a, b, r)+1$.

5. Algorithm is stopped when the accumulator array reaches a certain threshold value. The next step is to search for the peaks in the accumulator array.

\subsection{Pseudo code for $\mathrm{RCHT}$}

1. First, digitize the original image.

2. Initialize the accumulator array to zero.

3. Save the values of the image pixel to a data set $P$.

4. Randomly pick three points from set $P$.

5. Set up a threshold value. For each pixel passes the certain threshold value calculate the value for radius $r$, for all possible values of $a$ and $b$.

6. Then increment the accumulator array.

7. end.

In order to achieve computational time efficiency, wavelet based downsampling technique was applied. The downsampling factor (defined as inc in the RCHT implementation) in this case was found as 15 . This factor was chosen in such a way that both computational time and error reduction could be balanced. For downsampling values less than 15, the process takes more than half a day to compile. For values greater than 15, more error is introduced. 


\subsection{Simulation Results}

Using steps mentioned above, RCHT was applied on a circular image of size $185 \times 185$ pixels.

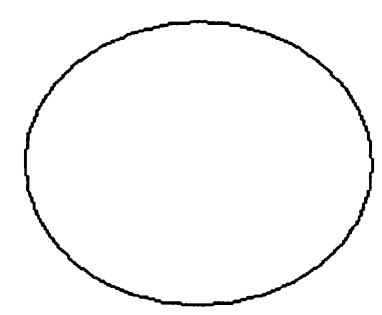

Figure 4.1: 185x185 pixels sample circular image

For this image, the parameters $r$, $a$ and $b$ are found as $r=82, a=6 *_{\text {inc }}=90$ and $b=7 *$ inc $=105$. For this image, the values for $r$, $a$ and $b$ were found out by using the 'ginput' command. These values are, $r=76.9, a=94.5$ and $b=94.5$. While comparing program outputs and the original parameters of the circle, it can be observed that from RCHT, the parameters of the circle were detected with an accuracy of more than 90 percent.

It is clear that any parametric shape could be optimally processed by $\mathrm{HT}$ and it is typical in many signal/image processing applications to detect and recognize information carrying arbitrary shapes. The next chapter talks about applying HT for arbitrary shapes. 


\section{Chapter 5}

\section{RGHT For arbitrary shapes}

\subsection{RGHT For arbitrary shapes}

It is well known that RHT works well for features with parametric equation. In case of features which lack a mathematical equation we have to fit a polynomial to get the coefficients. RGHT was implemented using the Newton's curve fitting technique. Newton's general interpolating formula for data pairs $(x 1, y 1)$ through $(x n, y n)$ has the form

$$
y=a 0+a 1 *(x-x 1)+a 2 *(x-x 1) *(x-x 2)+\ldots \ldots .
$$

where $a 0, a 1, \ldots$ are the coefficients. When considering the computational time involved in this process, only the coefficients $a 0, a 1, a 2$ were taken into consideration and the rest of the coefficients were ignored. In this case, the accumulator array is five dimensional which is $A(a 0, a 1, a 2, x 1, x 2)$. By rearranging this equation to solve for $a 0$, we get

$$
a 0=y-a 1 *(x-x 1)-a 2 *(x-x 1) *(x-x 2)
$$

Before computing the RGHT of any curve, the parameter space between appropriate maximum and minimum values of a0min and $a 0 \mathrm{max}, a 1 \mathrm{~min}$ and $a 1 \max , a 2 \min$ and $a 2 \max , x 1 \min$ and $x 1 \max$, and $x 2 \min$ and $x 2 \max$ should be quantized. Once quantization of the parameter space is done, 
the actual HT method can be implemented. The procedure is the same as adopted for straight line except the dimensionality is high as compared to the straight line problem.

The algorithm used to implement RGHT for Arbitrary Shapes is given below:

1. Scan the image and accumulate the image points in set $P$.

2. Initialize accumulator array vote $(a 0, a 1, a 2, x 1, x 2)=0$.

3. Pick 5 points randomly from the set $P$.

4. For each point $(x i, y i)$ vary $a 1$ and $a 2$ from 1 to the number of rows in the image, also vary $x 1$ and $x 2$ from 1 to the number of columns in the image.

5. Next, in the limited quantized plane, increment the number of counts each time a point in the arbitrary shape passes through each cell until the transformation is completed. The number of counts in each cell of the parametric image are called votes, $A(a 0, a 1, a 2, x 1, x 2)=$ $A(a 0, a 1, a 2, x 1, x 2)+1$.

6. Local maximum in the accumulator array corresponds to collinear points in the image plane. The accumulator array provides a measure of the number of points on the arbitrary shape.

7. Algorithm is stopped when the accumulator array reaches a certain threshold value. The next step is to search for the peak in the accumulator array. The peaks and their corresponding coordinates are used to identify potential shapes within the image plane. These peaks provide the values of $a 0, a 1, a 2, x 1$ and $x 2$. 


\subsection{Pseudo code for RGHT for Arbitrary Shape}

1. First, digitize the original image.

2. Initialize the accumulator array to zero. Save the values of the image pixel to a data set $P$.

3. Set up a threshold value. For each pixel passes the certain threshold value calculate the coefficient $a 0$ for all possible values of $a 1, a 2, x 1$ and $x 2$. The formula to calculate $a 0$ is given below: $\mathrm{a} 0=\mathrm{y}-\mathrm{a} 1^{*}(\mathrm{x}-\mathrm{x} 1)-\mathrm{a} 2^{*}(\mathrm{x}-$ $\mathrm{x} 1)^{*}(\mathrm{x}-\mathrm{x} 2)$.

4. Then increment the accumulator array.

5. end.

\subsection{Analysis of Results}

Using steps mentioned above, RGHT was applied on an arbitrary shape (See figure 5.1). The parametric equation of this arbitrary shape is $y=a o+a 1 *(x-x 1)+a 2 *(x-x 1) *(x-x 2)=1+1 *(x-1)+1 *(x-1) *(x-1)$

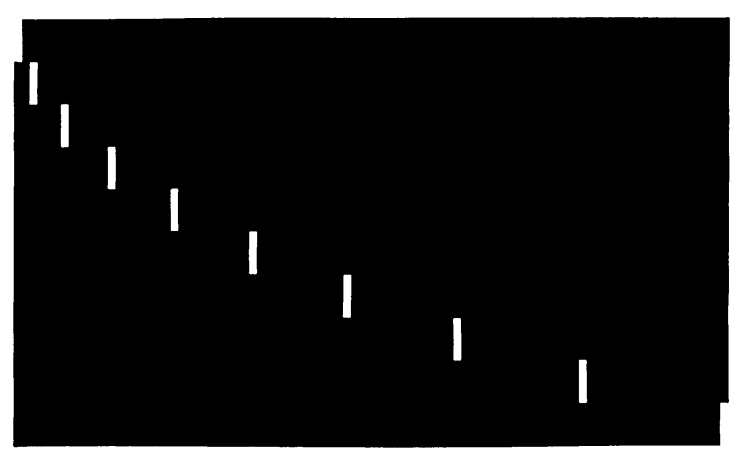

Figure 5.1: An arbitray shape represented by the mathemaical equation $y=a o+a 1 *(x-x 1)+$ $a 2 *(x-x 1) *(x-x 2)=1+1 *(x-1)+1 *(x-1) *(x-1)$ 
From the RGHT program output, the maximum vote was found as 5 points located at $a 0=7, a 1=4, a 2=3, x 1=5$ and $x 2=2$. Since the original equation of the arbitrary shape is

$$
y=1+1 *(x-x 1)+1 *(x-1) *(x-1)=x^{2}-x+1
$$

Since a2 denotes the coefficient of the highest order term in the above eqn, a2 determines the characteristics of the shape. From the output of the GHT, the equation of the arbitrary shape is obtained as

$$
y=7+4 *(x-3)+1 *(x-3) *(x-2)=x^{2}-x+1
$$

which agrees with the original equation of the image as in equation (4.2). Also note that $a 2$ value for the original image as well as the $a 2$ value obtained from the Basic RGHT are the same, thus confirms the accuracy of the result.

Since the accumulator array is five dimensional for RGHT, computational complexity is very high. In order to achieve computational time efficiency, Wavelet decomposition technique was applied on the image. RGHT algorithm was applied on the decomposed version of the image and the value of $a 0, a 1, a 2, x 1, x 2$ are found as $a 0=3, a 1=3, a 2=1 x 1=2, x 2=2$ Therefore, the equation of the arbitrary shape is found as

$$
3 *(x-2)+1(x-2) *(x-2) .
$$

After simplification, the equation (5.4) becomes, $y=x^{2}-x+4$ which agrees with the equation of the original image, except for the intercept.

\subsection{HWT for Arbitrary Shapes}

Since the accumulator array is five dimensional for RGHT, computational complexity is very high. In order to achieve computational time efficiency, Wavelet decomposition technique was applied on the image. RGHT algorithm 
was applied on the decomposed version of the image. The following table gives a comparison of the RGHT results before and after the decomposition.

\begin{tabular}{|c|c|c|}
\hline & RGHT without decomposition & RGHT with level 1 Decomposition \\
\hline Time (sec) & 3.133 & 0.8907 \\
\hline
\end{tabular}

Table 5.1: RGHT time comparison table before and after decomposition

It can be observed that the decomposition process considerably improved the time efficiency of RGHT. Time efficiency achieved in this case was more than 78

\subsection{Image Enhancement}

In this project, the theory of minimum cross entropy optimization has been applied for image enhancement purposes and also to achieve time efficiency. In the crude image,there were 4 less intensity points and the intensity of them were 150 each.After applying the newly developed MCE optimization coding, it was found out that 3 out of 4 of the less intensity points became significantly enhanced with intensity values of 238, 238 and 405 and thus confirms MCE's applicability in image enhancement. RSLHT was applied on the crude image and also on the enhanced image and the results were compared. The following table gives a comparison of the RSLHT results before and after the enhancement.

\begin{tabular}{|c|c|c|}
\hline & Crude Image & Enhanced Image \\
\hline Maximum vote & 3 & 3 \\
\hline Coordinate(a0,a1,x1) & $(50,1,50)$ & $(50,1,50)$ \\
\hline Time $(\mathrm{sec})$ & 0.1646 & 0.0987 \\
\hline
\end{tabular}

Table 5.2: RSLHT Results 
From table 4.2, it can be noted that considerable time efficiency was achieved when applying RLSHT on an enhanced image.

\subsection{Observations}

It can be observed from the RGHT results that the coefficients of a0, a1 and $\mathrm{x} 1$ obtained from the first level of wavelet decomposition are about half the value of the coefficients obtained from the Basic RGHT. However, the value of coefficients a 2 and $\mathrm{x} 2$ remain the same before or after applying the wavelet decomposition. In conclusion, after applying the wavelet decomposition, the coefficients of the image maintain a 50 percent ratio while comparing to the real coefficients before applying the decomposition technique.

\subsection{Performance}

The RGHT using the proposed method proved as a very efficient technique for the detection of complex shapes. The new technique was able to detect any arbitrary shape. Since the dimensionality of the accumulator array was high, without applying wavelet decomposition technique, it was almost impossible to perform RGHT. Also, enhancing the image(before applying the HT) using the MCE technique, proved to accomplish the required time efficiency. 


\section{Chapter 6}

\section{Computational Complexity of Proposed Method}

\subsection{Computational Complexity of Proposed Method}

Computational complexity of RGHT is very less compared to other HT types. Random sampling mechanism and the converging mechanism of RGHT helps it to be the best computationally attractive technique among other HT types. The following table provides a detailed analysis of this.

\begin{tabular}{|c|c|c|c|c|}
\hline Algorithm & HT & GHT & RHT & RGHT \\
\hline \multicolumn{5}{|c|}{ image type- } \\
\hline Binary & yes & yes & yes & yes \\
\hline Grayscale & no & yes & no & yes \\
\hline \multicolumn{5}{|c|}{ target- } \\
\hline line & yes & no & yes & yes \\
\hline circle & yes & yes & yes & yes \\
\hline parametric & yes(slow) & yes & yes & yes \\
\hline Arbitrary & no & yes & no* & yes \\
\hline Speed & slow & slow & fast & fast \\
\hline Storage & high & high & low & low \\
\hline Accuracy & medium & medium & high & high \\
\hline Resolution & low & low & any & any \\
\hline
\end{tabular}

Table 6.1: Comparison between RGHT and other Hough Transforms 
* RHT itself is not suitable for arbitrary shape detection. In order to make this possible, a mathematical way of representing any arbitrary shape has to be developed.

1 RHT itself is not suitable for grayscale images. In order to make RHT suitable for grayscale images, thresholding or filtering has to be applied. The following table provides an illustration of the Time Complexity of HT related Algorithms.

\begin{tabular}{|c|c|}
\hline Algorithm & Time of Complexity \\
\hline HT & $\mathrm{N} / \mathrm{A}$ \\
\hline GHT & $\mathrm{O}\left(X_{\text {size }} \cdot Y_{\text {size }}\right)$ \\
\hline RHT & $\mathrm{O}($ number of sample $\cdot f(n))$ \\
\hline RGHT & $\mathrm{O}($ number of sample $\cdot f(n))$ \\
\hline
\end{tabular}

Table 6.2: Time Complexity of HT related Algorithms

Since HT is unable to detect arbitrary shapes, the time complexity of HT is not included here. In RHT, $\mathrm{f}(\mathrm{n})$ is a function which depends on the number of parameters $\mathrm{n}$ of the analytical object. Since there are two parameters involved in the case of a line, $\mathrm{f}(\mathrm{n})=n^{2}$. Also number of sample is the number of pixels which are picked randomly in a specific image which is $\mathrm{n}$. Therefore, in the case of a straight line, the time complexity is found as $n^{3}$. Since newly proposed RGHT follows the same concept as in the case of RHT, the time complexity equation will be same. But the only difference is that since 3 parameters are involved in the proposed method in the case of line detection, $\mathrm{f}(\mathrm{n})$ will be $n^{3}$ and therefore the time complexity of the proposed method is $n^{4}$. 


\subsection{Potential Applications of RGHT}

This promising technique could find many practical applications in the area of Spectroscopy, Pattern Recognition, Machine Vision and also in several medical areas. One such application is the detection of Echelle disorders. This technique also can be used for the detection of linear/non-linear chirp interferences in a spread spectrum signal [15] .

\subsection{Conclusion}

The RGHT using the proposed method proved as a very efficient technique for the detection of complex shapes. The new technique was able to detect any arbitrary shape. Since the dimensionality of the accumulator array was high, applying the wavelet decomposition technique, helped the process to become faster. Also MCE's applicability on image enhancement was also proven effective in this project. After applying RGHT on the enhanced image proved much more time efficient than applying RGHT on the crude image itself. 


\section{Bibliography}

[1] D.H. Ballard, Generalizing the Hough Transform to Detect Arbitrary Shapes, Pattern Recognition, Vol.13, No.2, pp. 111-112, 1981.

[2] R.O. Duda and P.E. Hart, Use of the Hough Transform To Detect Lines and Curves in Pictures, Stanford Research Institute Menol Park, California, Volume 15, Number 1, pp. 11 -14, January 1972.

[3] J. Illingworth and J. Kittler, A Survery of the Hough Transform, Computer Vision, Graphics, and Image Processing, Volume 44, pp. 87 -116, 1988.

[4] S. Thayilchira, An efficient image analysis using Hough and Wavelet Transforms, BEng Thesis report, Ryerson University, 2001.

[5] P. Kultanen, L. Xu, and E. Oja, Randomized Hough Transformation (RHT), Pattern Recognition Letters, pp. 331-338, 1990.

[6] P. Fung, W.S. Lee, and I. King, Randomized generalized Hough Transform for 2-D grayscale object detection, Pattern Recognition, 1996, Vol. 2, pp. 511-515.

[7] P. Kultanen, L. Xu, and E. Oja, Randomized Hough Transform (RHT): Theoretical Analysis and Extensions, Research Report 18, Lappeenranta University of Technology, Department of Information Technology, Lappeenranta, Finland, 1990. 
[8] P. Kultanen, L. Xu, and E. Oja, Generalized Randomized Hough Transform (GRHT) , Research Report 20, Lappeenranta University of Technology, Department of Information Technology, Lappeenranta, Finland, 1990.

[9] X. Lei, H. Kalviainen, P. Hirvonen, and E. Oja Probabilistic and nonprobabilistic Hough transforms: overview and comparisons, Image and Vision Computing, Volume 13, Number 4, pp. 239-252, May 1995.

[10] Krishnan et al., Adaptive Time-Frequency Analysis of Knee Joint Vibroarthrographic Signals for Noninvasive Screening of Articular Cartilage Pathology, IEEE Transactions On Biomedical Engineering, vol. 47, no. 6 , JUNE 2000.

[11] P.J. Loughlin, J.W. Pitton, and L.E. Atlas, Construction of Positive Time-Frequency Distribution, IEEE Transactions on Signal Processing, Vol. 42, NO. 10, pp. 2697-2705, 1994.

[12] P. J. Loughlin, Time-Frequency energy density function: theory and synthesis, Ph.D Dissertation, Univ. of Washington, 1992.

[13] V. Kamat-Sadekar and S. Ganesan, Complete description of multiple line segments using the Hough Transform, Image and Vision Computing, Vol. 16, pp. 597-613, 1998.

[14] P. Ballester, Application of the Hough Transform, Astronomical Data Analysis Software and System III, ASP Conference services, Volume 61, 1994.

[15] S. Thayilchira and S. Krishnan, Detection of linear and non-linear chirps in spread spectrum signals using Hough-Radon Transform, Proc. International Conference on Acoustics, Speech, and Signal Processing (ICASSP), Orlando, FL., USA, May 2002. 
[16] T.J.Akai, Applied Numerical Methods for Engineers, John Wiley and Son Inc., New York, 1994.

[17] S. Thayilchira and S. Krishnan, Generalized Hough Transform, International Conference for Upcoming Engineers (ICUE), Toronto, Ontario, 2001.

[18] David Young, Hough Transform, January 1993, Volume 16, pp. 597-613 http://www.cogs.susx.ac.uk/users/davidy/teachvision/vision4.html (Accessed on November 1, 2006).

[19] Robi Polikar, The Engineer's Ultimate Guide to Wavelet Analysis, http://www.public.iastate.edu/rpolikar/WAVELETS/WTtutorial.html (Accessed on November 1, 2006).

[20] John E. Shore and Rodney W. Johnson, Axiomatic Derivation of the Principle of Maximum Entropy and the Principle of Minimum CrossEntropy, IEEE Transactions on Information Theory, Vol. IT-26, NO.1, January 1980.

[21] M. Soffer and N. Kiryati, Guaranteed Convergence of the Hough Transform, Computer Vision and Image Understanding, Vol. 69, NO. 2, pp. 119-134, February 1998.

[22] John E. Shore and Rodney W. Johnson, Properties of Cross-Entropy Minimization, IEEE Transactions on Information Theory, Vol. IT-27, NO.4, July 1981.

[23] J. Bigun, Coordinate Transformations, Symmetries, and GHT, International Conference on Image Processing, Volume 3, pp. 197-201, September 1996 . 
[24] J. P. Noonan and E. Laderman, Minimum Cross Entropy Spectral Estimation Using Non-Linear Optimization, Proceedings of the IEEE International Symposium on Circuits and Systems, San Diego CA, May 1989.

[25] N. C. Fitton and S. J. D. Cox, Optimising the application of Hough Transform for automatic feature extraction from geoscientific images, Computers and Geosciences, Volume 24 , Issue 10, pp. 933 - 951, December 1998.

[26] C. M. Brown, M. B. Curtiss, and D.B. Sher, Advanced Hough Transform Implementations, International Joint Conference on Artificial Intelligence (IJCAI83), pp. 1081-1085, 1983. 